\title{
Coordinate of Unicycle Retention Return Decade Resting Phase
}

\author{
Masami Miyazaki ${ }^{*}$ Takeshi Sato ${ }^{* \dagger}$ \\ $\begin{array}{ccc}\text { Masami Miyazaki } & \text { Takeshi Sato } & \text { Takayuki Watanabe } \\ \text { Kazuyoshi Seki }^{*} & \text { Eiji Watanabe } & \text { Nobuaki Kawai }^{*}\end{array}$ \\ (*)Waseda University, Japan \\ (*))Jissen Women's University, Japan \\ (†)Hachinohe University, Japan \\ E-mail:mayazaki@waseda.jp,sato-takeshi@jissen.ac.jp,nabetaka@hachinohe-u.ac.jp, \\ kkazu@waseda.jp,watana@aoni.waseda.jp,kawai-nobuaki@jissen.ac.jp,
}

\begin{abstract}
Analysis of motor skill and performance variability in task with retention affords insight about synergies underlying central nervous system control. Preferential distribution of variability in way that minimally affected the task performance suggests sophisticated neural control. In around 1990, it was adopted monocycle exercise as an educational program in all over the Japanese elementary school. Unfortunately, it was not used the monocycle in daily life. This research assesses the influence of coordinate in motor skills retention. It was measured Electromyography and accelerometer during monocycle motor tasks for ten or more year break interval. It was rapidly activated monocycle performance in all subjects. An analysis of variability in execution using motor retention to quantify performance at results offers substantially more sensitivity to coordinated variables in motor tasks.
\end{abstract}

\section{Introduction}

A study of multivariable behavior naturally raise the question of which reference frames the central nervous system may use to coordinate motor actions. Previous studies were focused planar reaching movements and showed that translation and rotation of the start and target positions evoked joint kinematics [1]. This investigation of the retention at highly skilled motor performance about unicycle task, electromyography (EMG) activity by human leg muscles was prompted by findings from locomotor behavior. Moreover, it was applied unicycle educational program for acquired physical sense of balance in Japanese elemental school. However it was not well known to acquire the motor skill in unicycle and not enough data of its retention. The purpose of this study was to investigate of retention skills in unicycle from over 10 years resting period.

\section{Method}

\subsection{Participants}

Seven female university students with a mean \pm SD age, stature, body mass and BMI of $21.2 \pm 1.0$ years, $158.0 \pm 5.0 \mathrm{~cm} 48.0 \pm 9.5 \mathrm{~kg}$ and, $19.1 \pm 1.9$, respectively, volunteered to participate in the study. Experimental procedures were reference primary school's textbook. The protocol was explained and subjects gave the informed consent prior to the participation in this study. All subjects were physically active in additionally they had experiment with riding the unicycle in childhood, and had abstained from alcohol, caffeine and exercise for $24 \mathrm{~h}$ preceding the investigation to ensure reliability.

\subsection{Experimental settings}

Following several warm up physical activity except for riding any cycle, participants were performed riding unicycle for the first time in a minimum decade. They had tried the unicycle back-to-back until $10 \mathrm{~m}$ for time test by 10 times in a same day. Also it was put on the electrode of the EMG and sensor of three dimensions accelerometer on top of head was recorded into the PC for later analysis.

\subsection{Electromyography and Accelerometer}

Surface EMG activity of the rectus femoris (RF) in both left and right was recorded while performing unicycle riding using pre-gelled two $10-\mathrm{mm} \mathrm{Ag}-$ AgClunipolar electrodes. Electrodes were placed along the mid-sagittal axis of the muscle. Prior to the

This is an Open Access article distributed under the terms of the Creative Commons Attribution-Noncommercial License 3.0, which permits unrestricted use, distribution, and reproduction in any noncommercial medium, provided the original work is properly cited. 
placement of the electrodes, the skin was shaved to remove the hair and the recording sites were gently rubbed using abrasive gel and cleansed using alcohol swabs to reduce inter-electrode impedance below $5 \mathrm{~K}$. And the sensor device of three dimensional accelerometly put on top their helmnet (Figure 1). The raw EMG activity and Accelerometer was acquired with a sampling frequency of $2 \mathrm{kHz}$ to data logger in participant's backpack and processed with a multichannel analogue-digital converter. Statistical processing used one way repeated measures of ANOVA $(=0.05)$.

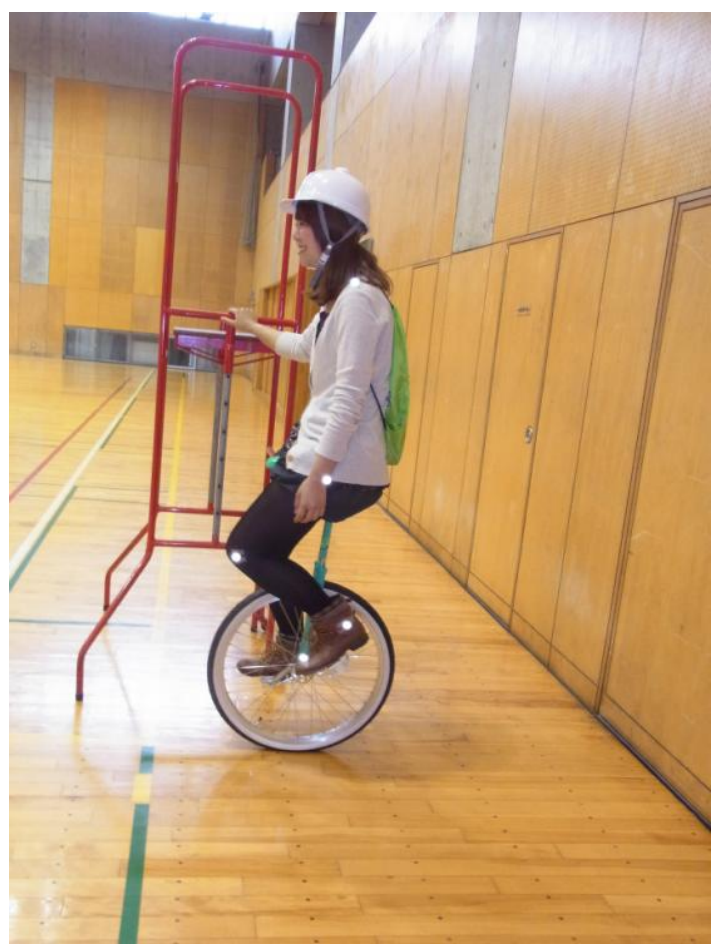

Figure 1. Experimental setting on unicycle. Participants performed to riding $10 \mathrm{~m}$ time trial by 10 times, with handle onset. For safety's sake, experimenter was right by side without their eyesight.

\section{Result}

Three participants were not reached by their own dropping the unicycle unless $2 \mathrm{~m}$ line from onset each first trial. The overall effect of retention on the average EMG activity was consistent, and it may be seen typical data collection. It was decreased $10 \mathrm{~m}$ riding performance time test on unicycle from 7.86 to 4.95 seconds (Figure 3). There were decreased gradually sway Y-axis from first trial to end trial. Instead smaller sway movements, it was increased $\mathrm{X}$-axis movement of running to forward daynamically (Figure 4). From the integrated EMG signals recorded during the unicycle riding $10 \mathrm{~m}$, no statistical differences were observed throughout the investigation.

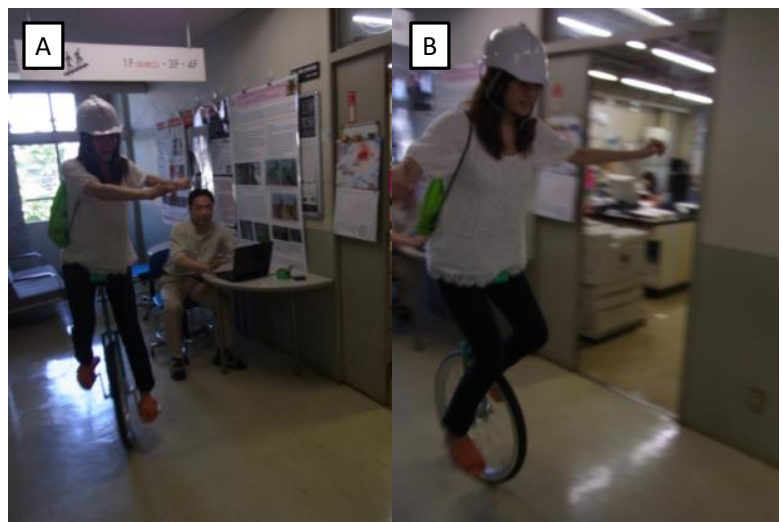

Figure 2. A: First riding on unicycle from a decade break period. B: Fifth trial, extended both participant's arms..

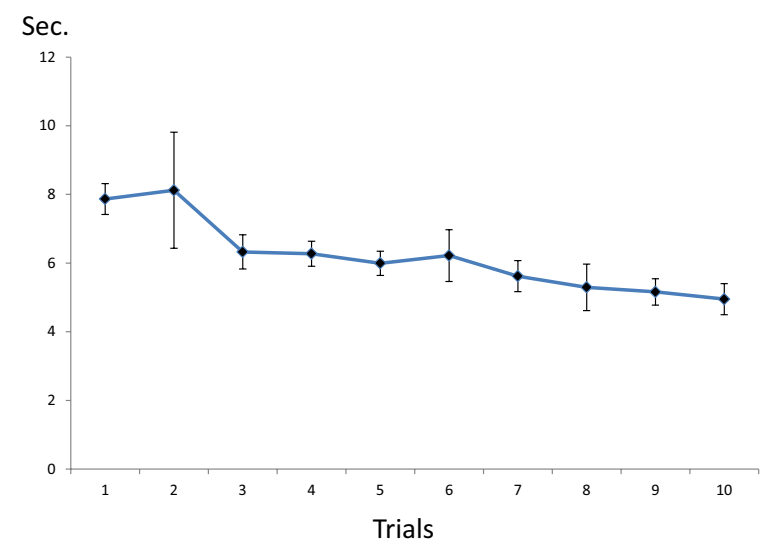

Figure 3. Average time test in 10times trials.

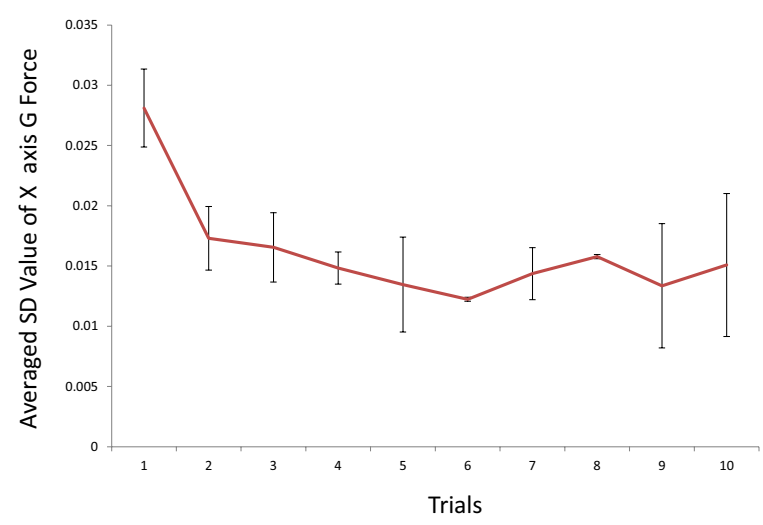

Figure 4. Averaged Standard Deviation value in $\mathrm{X}$ axis (Front Face direction) Accelerometer( $G$ force) data in each trials. 


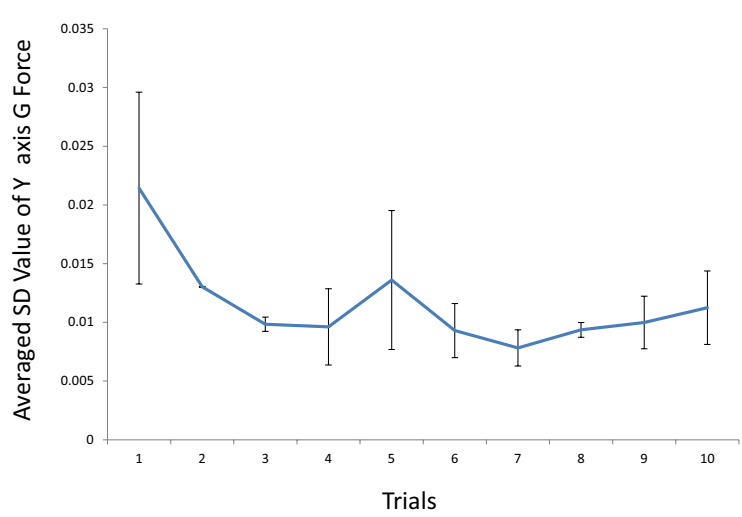

Figure 5. Averaged Standard Deviation value in $\mathrm{X}$ axis (horizontal direction) Accelerometer( $\mathrm{G}$ force) data in each trials.

Figure 5 was showed that it was decreasing the value of standard deviation of horizontal accelemeter. It was observed without wooziness with riding unicycle.

\section{Discussion}

We examined muscle activation and three dimensions accelerometer in unicycle riding, major found that the retention has been acquired in all subjects. However the first or second trial especially swayed more than continued trails. It was appeared that once acquired motor skill was retention as same as swimming, chopsticks and bicycle riding. However after first two trials, it was increased riding speed dramatically. Futuremore, it was necessary to evaluate the educational skill of unicycle, it was affected to any daily life in human movement. Consequently it was essential 2 trials to activated retention from a decade rest period. Approximately over 5 times trials, participant were to effort time test in their own skills, therefore there were motor control skill between $\mathrm{X}$ axis ( going ahead quickly) and $\mathrm{Y}$ axis ( moving stable riding), respectively.

\section{References}

[1] Morasso P, Spatial control of arm movement, Exp Brain Res, 42, pages. 223-227, 1981 